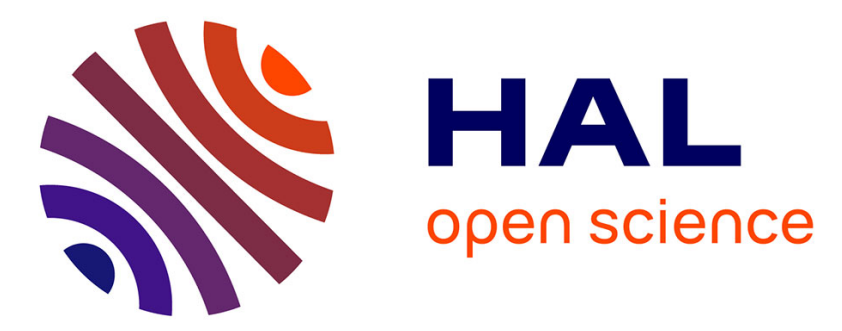

\title{
Métropoles des pays émergents : des acteurs de la transition énergétique? Leçons du Cap (Afrique du Sud)
}

Sylvy Jaglin

\section{To cite this version:}

Sylvy Jaglin. Métropoles des pays émergents: des acteurs de la transition énergétique? Leçons du Cap (Afrique du Sud). Géographie, Économie, Société, 2017, 19 (2), pp.243-265. 10.3166/ges.19.2017.0012 . hal-01497585

\section{HAL Id: hal-01497585 \\ https://hal.science/hal-01497585}

Submitted on 23 Apr 2018

HAL is a multi-disciplinary open access archive for the deposit and dissemination of scientific research documents, whether they are published or not. The documents may come from teaching and research institutions in France or abroad, or from public or private research centers.
L'archive ouverte pluridisciplinaire HAL, est destinée au dépôt et à la diffusion de documents scientifiques de niveau recherche, publiés ou non, émanant des établissements d'enseignement et de recherche français ou étrangers, des laboratoires publics ou privés. 


\title{
MÉTROPOLES DES PAYS ÉMERGENTS : DES ACTEURS DE LA TRANSITION ÉNERGÉTIQUE ? LEÇONS DU CAP (AFRIQUE DU SUD) Sylvy Jaglin
}

\author{
Lavoisier | "Géographie, économie, société »
}

2017/2 Vol. 20 | pages 243 à 265

ISSN 1295-926x

ISBN 9782743023119

Article disponible en ligne à l'adresse :

http://www.cairn.info/revue-geographie-economie-societe-2017-2-page-243.htm

\section{Pour citer cet article :}

Sylvy Jaglin, « Métropoles des pays émergents : des acteurs de la transition énergétique ? Leçons du Cap (Afrique du Sud) », Géographie, économie, société 2017/2 (Vol. 20), p. 243-265.

DOI 10.3166/ges.19.2017.0012

Distribution électronique Cairn.info pour Lavoisier.

(C) Lavoisier. Tous droits réservés pour tous pays.

La reproduction ou représentation de cet article, notamment par photocopie, n'est autorisée que dans les limites des conditions générales d'utilisation du site ou, le cas échéant, des conditions générales de la licence souscrite par votre établissement. Toute autre reproduction ou représentation, en tout ou partie, sous quelque forme et de quelque manière que ce soit, est interdite sauf accord préalable et écrit de l'éditeur, en dehors des cas prévus par la législation en vigueur en France. Il est précisé que son stockage dans une base de données est également interdit. 


\title{
Métropoles des pays émergents : des acteurs de la transition énergétique? Leçons du Cap (Afrique du Sud)
}

\author{
Sylvy Jaglin \\ Université Paris-Est, Latts ENPC \\ 6 et 8 avenue Blaise Pascal - Cité Descartes \\ 77455 Marne-La-Vallée Cedex 2
}

\section{Résumé}

De manière croissante, les villes sont considérées comme des lieux et des "acteurs" stratégiques de la transition énergétique. Pour tester la robustesse de ce constat dans quatre métropoles de pays émergents, la recherche collective Termos est partie de deux questions : dans quelle mesure les protagonistes locaux urbains sont-ils acteurs d'une transition énergétique urbaine et laquelle? Dans quelle mesure ce mouvement s'accompagne-t-il d'une territorialisation urbaine des systèmes énergétiques? L'article présente d'abord les conclusions de cette recherche comparative, qu'il prolonge ensuite par l'exemple plus détaillé du Cap. Il analyse pourquoi, en dépit de son volontarisme énergétique et environnemental, la municipalité n'a que peu de marges de manœuvre tandis que les changements observés semblent renforcer la mainmise de la «sphère centrale » de pouvoir sur le système énergétique. Appréhendant cette situation comme l'expression d'un conflit entre un puissant secteur électrique national et une approche alternative des questions énergétiques portée par des acteurs urbains, il souligne les tensions qui en résultent et leurs conséquences sur les engagements de la municipalité, à la fois limités par des résistances mais aussi «phagocytés » par des acteurs de la sphère centrale. Il revient, pour finir, sur les enseignements de cet exemple pour l'analyse générale des dynamiques observées dans d'autres villes de pays émergents.

(c) 2017 Lavoisier, Paris. Tous droits réservés

Mots clés : transition énergétique, secteur électrique, énergies renouvelables, pays émergents, Le Cap, Afrique du Sud.

Adresse mail : jaglin@enpc.fr doi : 10.3166/ges.19.2017.0012 @ 2017 Lavoisier, Paris. Tous droits réservés. 


\begin{abstract}
Summary
Metropolises in emerging countries: actors in energy transitions? Lessons from Cape Town (South Africa). The role of cities, as places and drivers of the energy transition is increasingly recognized. The research project Termos tested the robustness of this assumption in four cities of emerging countries by asking two questions: to what extent do urban local actors really drive an urban energy transition and of what kind? To what extent are their actions supporting an urban territorialisation of energy systems? The paper first presents the findings of this comparative research, which he then extends with the case study of Cape Town. It analyzes why, despite their energy and environmental voluntarism, the municipality have little room of manoeuvre, while the changes observed seem to strengthen the stranglehold of the "central sphere» in the energy system. Analyzing this as the expression of a conflict between a strong national electricity sector and an alternative approach to energy issues carried by urban actors, it highlights the resulting tensions and their impact on the municipal actions, both limited by resistance but also «swallowed up» by actors from the central sphere. The paper finally draws lessons from this example to enrich the general analysis of dynamics observed in other cities of emerging countries.

(c) 2017 Lavoisier, Paris. Tous droits réservés
\end{abstract}

Keywords: energy transition, electricity sector, renewable energies, emerging countries, Cape Town, South Africa.

\title{
Introduction
}

Dans les débats internationaux, l'expression générique de transition énergétique qualifie une transformation délibérée du système énergétique vers un modèle moins carboné, reposant sur un mix diversifié plus ouvert aux énergies renouvelables et promouvant une plus grande sobriété (Rojey, 2008) ${ }^{1}$. Elle est en outre souvent associée, voire conditionnée, à une relocalisation de la chaîne énergétique susceptible de limiter les facteurs d'incertitude et de faciliter la construction d'écosystèmes territoriaux résilients (Durand et al., 2015). Cette relocalisation serait une condition favorable, voire nécessaire, pour développer une approche désectorisée des questions énergétiques, combinant des logiques d'offres, multiples et complémentaires, avec des approches de gestion de la demande et d'efficacité énergétiques.

Si les villes, qui regroupent plus de la moitié de la population mondiale, ne sont pas les seuls territoires concernés par cette relocalisation, elles sont néanmoins considérées comme des lieux et des acteurs stratégiques de la transition énergétique (Bulkeley et al., 2011; Hodson et Marvin, 2010). Dès 1999, Capello et al. écrivaient ainsi « Modern cities can indeed play a strategic role in the necessary pathway to sustainable development, with particular emphasis on the opportunities offered by local energy and environmental initiatives » (p. v). Depuis, ce constat a été à la fois discuté, précisé et nuancé par des travaux qui, portant principalement sur des villes de pays dits « développés », anticipent néanmoins une montée en puissance des acteurs locaux urbains dans la gouvernance énergétique (Buckeley et al. 2014; Rutherford et Coutard, 2014; Monstadt et Wolf, 2015; Rutherford et Jaglin, 2015; Theys et Vidalenc, 2013; Thorp et Marvin, 1995).

\footnotetext{
${ }^{1}$ La définition de la transition énergétique n'est cependant pas sans ambiguïté ni débats : voir par exemple Les Cahiers de Global Chance, $\mathrm{n}^{\circ} 35$, juin 2014, http://www.global-chance.org/Autour-de-la-transitionenergetique-questions-et-debats-d-actualite.
} 
Pour tester cette hypothèse dans des métropoles de pays émergents, la recherche collective Termos s'est intéressée aux facteurs de changement observables dans quatre villes ${ }^{2}$ et posait deux questions : dans quelle mesure les protagonistes locaux urbains sont-ils acteurs d'une transition énergétique urbaine et laquelle? Dans quelle mesure ce mouvement s'accompagne-t-il d'une territorialisation urbaine des systèmes énergétiques? La première interroge l'existence d'une «vision » alternative des villes en matière de transition énergétique et de son contenu. La seconde porte sur la nature et l'ampleur d'un mouvement de rescaling des systèmes énergétiques, conférant un poids croissant aux enjeux urbains dans les décisions qui gouvernent ces systèmes, un rôle accru des autorités locales urbaines dans leur gouvernance et une intégration stratégique des parties décentralisées du système énergétique dans leur économie politique (Jaglin et Verdeil, 2013). "A focus on the way energy systems are territorialized draws attention to the different scales and arenas of political action that govern energy systems" (Bridge et al. 2013 : 336) : qualifiant les mutations de la « socio-geography of energy landscapes $»^{3}$, la notion de territorialisation urbaine englobe ainsi, d'une part, les redimensionnements techniques et spatiaux des systèmes énergétiques, d'autre part, les déplacements des pouvoirs de décision et de régulation au sein des configurations héritées au profit des autorités urbaines. Termos montre que, dans les villes étudiées, la transition énergétique n'apparaît pas structurante des politiques locales et ne s'accompagne pas d'une territorialisation de la gouvernance énergétique aux échelles métropolitaines (Jaglin et Verdeil, 2013).

Partant des conclusions de cette recherche comparative, l'article met ensuite en perspective et en discussion les résultats de l'étude conduite au Cap par l'auteur. Il cherche à comprendre pourquoi, en Afrique du Sud, les changements observés semblent renforcer la mainmise de la «sphère centrale » de pouvoir ${ }^{4}$ sur le système énergétique. L'exemple est d'autant plus intéressant que la municipalité, City of Cape Town (CoCT), est très active sur le front du développement durable et du changement climatique, qu'elle a très tôt pris des engagements politiques dans le domaine énergétique et qu'elle bénéficie d'une image positive reconnaissant son activisme vert. Pourtant, la municipalité demeure un acteur marginalisé dans la gouvernance énergétique en général, électrique en particulier, et semble contrainte à un «attentisme intelligent » (Theys et Vidalenc, 2013). Comme le souligne Dubresson, « dans les conditions actuelles de fonctionnement du gouvernement multiniveaux et en raison du poids considérable des acteurs de la sphère centrale, elle ne peut pas être un acteur majeur de la production d'énergies renouvelables. En revanche, sa capacité d'innovation peut lui permettre d'inventer des modes d'action publique anticipant une éventuelle décompression autoritaire de la gestion centralisée du système énergétique national » (2013 : 44).

${ }^{2}$ Le projet Termos («Trajectoires énergétiques dans les Régions Métropolitaines des Suds »), financé par l'ANR (2011-2013) et coordonné au Latts par S. Jaglin, a principalement porté sur quatre métropoles : Buenos Aires, Delhi, Le Cap et Istanbul. Il a fait l'objet d'une synthèse (Jaglin et Verdeil, à paraître) et d'un numéro spécial de Flux coordonné par S. Jaglin et E. Verdeil (2013) et consacré aux études de cas issues du projet.

${ }^{3}$ Energy landscape is defined as "the constellation of activities and socio-technical linkages associated with energy capture, conversion, distribution and consumption" in which the "material landscapes [...] are the product of social processes and the outcomes of conflict and negotiation among different social groups" (Bridge et al., 2013: 335)

${ }^{4}$ La Constitution sud-africaine de 1996 définit trois sphères de gouvernement (centrale, provinciale, locale), autonomes mais interdépendantes. 
Dans une première partie, l'article rappelle les principaux résultats de la recherche Termos avant de présenter le cadre analytique, inspiré des travaux d'Evrard (Evrard 2013; 2014), proposé pour approfondir l'étude de cas consacrée au Cap. Dans ce cadre, les faibles marges de manœuvre de la municipalité sont appréhendées comme l'expression d'un conflit entre un puissant secteur électrique national et une approche alternative des questions énergétiques portée par des acteurs urbains. L'analyse de la situation sud-africaine, dans la deuxième partie, présente d'abord le secteur national de l'électricité et les voies qu'il emprunte pour promouvoir une transition énergétique sous contrôle, puis le volontarisme énergétique et environnemental affiché au Cap. Désectorialisée et territorialisée, cette approche alternative des questions énergétiques est toutefois étrangère au secteur électrique national, dont elle bouscule les représentations et approches dominantes. La troisième partie souligne les tensions qui en résultent et leurs conséquences sur les engagements de la municipalité, à la fois limités par des résistances de la sphère centrale mais aussi «phagocytés » et réappropriés par celle-ci. La conclusion revient sur les enseignements de cet exemple pour l'analyse des dynamiques observées dans d'autres villes de pays émergents.

\section{La résistible émergence d'une gouvernance énergétique urbaine}

Centré sur les services énergétiques à destination des consommateurs résidentiels, industriels et commerciaux (à l'exclusion des transports) ${ }^{5}$, Termos a mobilisé une approche comparative de quatre métropoles de pays émergents, qui ont pour caractéristiques communes une croissance démographique et économique élevée, une solide insertion dans les marchés globalisés, un environnement et un savoir-faire institutionnels consolidés. Leur demande énergétique est également forte et soutenue par la consommation industrielle, les investissements publics, l'aspiration des couches « moyennes » à une modernité associée aux avantages récemment acquis du confort, de l'équipement des logements et de la mobilité automobile. La démarche ${ }^{6}$ avait pour objectif d'identifier les principaux changements observables aux échelles métropolitaines en termes de services énergétiques (hors transport), sans exclure a priori une ressource (électricité, gaz, solaire thermique, pétrole lampant, charbon de bois...) ou un principe d'action (sécurité d'approvisionnement, mâ̂trise de la demande, efficacité énergétique), dès lors qu'ils apparaissaient structurants dans les interventions ou intentions déclarées de transformation.

\footnotetext{
${ }^{5}$ Les principaux usages concernés sont le chauffage, la climatisation, la cuisson, la réfrigération, la production d'eau chaude, l'éclairage (y compris l'éclairage public), l'alimentation des appareils électro-ménagers et informatiques, celle des dispositifs de sécurité et, dans les bâtiments industriels, celle des machines.

${ }^{6}$ La méthodologie a mobilisé un matériau empirique issu d'études de cas approfondies, qui ont permis de contextualiser et d'historiciser les changements actuels, et reposant sur des approches qualitatives (analyse de documents/textes de loi/données/discours/presse; enquêtes de terrain : entretiens semi-directifs avec les acteurs urbains et des systèmes énergétiques, visites d'installation, participation à des réunions...). Ce matériau a été organisé à partir d'une grille d'analyse commune composée de 3 volets principaux : les politiques publiques urbaines de l'énergie : genèse, mise en œuvre, contestations; les configurations sociotechniques de l'offre d'énergie et leurs conditions d'évolution (genèse, expansion, changements/crises); les facteurs géographiques, historiques et culturels façonnant les questions énergétiques locales. Enfin, une analyse comparée visant à identifier les principales régularités et à qualifier les spécificités a ensuite conduit à une synthèse dont s’inspire le présent article.
} 
Cette partie présente les principaux enseignements de cette recherche (voir aussi Jaglin et Verdeil, 2013) qu'elle prolonge ensuite par l'élaboration d'un cadre interprétatif inspiré par les conditions singulières qui prévalent au Cap, où la tension entre adaptation et rupture, deux visions politiques de la transition énergétique, est particulièrement prégnante.

\subsection{Termos : les enseignements d'une approche comparée}

En réponse à la question sur l'existence d'une « vision » urbaine de la transition énergétique, Termos apporte peu d'éléments tangibles : ses résultats n’ont révélé ni convergence vers un modèle de transition unique ni même aspiration commune à un tel modèle aux échelles urbaines. Les études de cas mettent en lumière trois principaux facteurs d'explication. En premier lieu, les autorités urbaines conservent un rôle marginal dans les systèmes techniques énergétiques, marqués par la prégnance des cadres politiques et institutionnels nationaux et celle des choix stratégiques des États. En deuxième lieu, au-delà des discours normatifs omniprésents en faveur de l'écologisation énergétique, les recherches n'ont pas permis d'identifier de réelles coalitions urbaines vertes (associant acteurs économiques et élites politiques, voire une société civile organisée) porteuses de projets politiques cohérents dans ce domaine. Aussi les profils énergétiques urbains restent-ils surdéterminés par des facteurs locaux et des dispositifs socio-techniques hérités (dépendants des sources d'énergie primaire dominantes, de la nature des bases économiques locales, des modes et échelles d'organisation des pouvoirs politico-administratifs, des pratiques locales de consommation, des cultures de la contestation...). En troisième lieu, l'expression locale des questions énergétiques est partout insérée dans des compromis construits autour de priorités hétérogènes d'aménagement et de développement économique urbains. Ce processus de construction locale des problèmes énergétiques n'est ni harmonieux, ni dénué de rapports de pouvoir ou de récupération politicienne, et il reflète des conceptions divergentes du rôle de l'énergie dans les politiques et le développement urbains.

Deux enseignements procèdent de ces constats : la transition énergétique, en tant que projet politique urbain, n'est nulle part structurante des politiques locales dans les villes étudiées; les préoccupations énergétiques locales ne procèdent pas d'un agenda partagé avec les instances nationales ou internationales. Lorsque les autorités urbaines défendent des choix énergétiques, elles ne le font pas avec le point de vue des acteurs sectoriels nationaux (compagnies d'électricité, ministère de l'énergie, régulateur national, etc.) mais à partir d'approches trans-sectorielles qui leur sont inspirées par des domaines d'intervention dont elles ont la charge : transport, planification urbaine, logement, gestion du parc immobilier municipal, pauvreté et précarité, etc. Leur approche est donc d'emblée transversale et, de ce fait, en contradiction avec les approches sectorielles des acteurs nationaux, notamment ceux en charge de l'électricité.

En conséquence, la territorialisation urbaine des politiques énergétiques n'a pas été non plus confirmée par les travaux de Termos, qui ont plutôt conduit à constater l'urbanisation croissante des questions énergétiques, dont toutes les études de cas illustrent diverses modalités. Par là, il faut entendre, d'une part, l'intégration croissante des questions énergétiques dans les politiques urbaines, et d'autre part, l'importance grandissante des discours, actions, conflits autour des questions d'énergie qui s'expriment dans les villes et influencent les changements énergétiques, même si ceux-ci sont commandés à d'autres échelles : plutôt qu'à une autonomisation des acteurs voire des intérêts urbains énergétiques, on assiste à leur 
prise en compte croissante dans la gouvernance énergétique d'échelle nationale. Cette prise en compte a des incidences notables : elle positionne les villes, notamment les plus grandes, comme de possibles interlocuteurs dans un jeu d'acteurs multiniveau, elle valorise leur rôle d'incubation ou d'entraînement des changements énergétiques mais elle contribue aussi à importer dans les systèmes énergétiques centralisés des demandes, des contestations et des résistances qui sont celles des consommateurs urbains.

L'énergie est au cœur de nombreux enjeux de développement économique local, d'aménagement urbain et d'équipement des logements, de consommation... Lorsque les autorités publiques municipales s'en emparent, c'est autour de quatre principales préoccupations dans les villes étudiées : les défaillances du service et la sécurité d'approvisionnement; les inégalités d'accès au service; la qualité environnementale (notamment la pollution de l'air); l'emploi et le développement local. Des leviers d'action existent donc aux échelles urbaines et sont parfois actionnés par les collectivités locales mais leurs interventions ne répondent pas d'emblée à un projet explicite de transition énergétique, même si elles peuvent aussi y concourir. Ainsi, face à l'insécurité d'approvisionnement, la diversification du mix énergétique est un recours que tente d'exploiter la municipalité du Cap. Lorsque des politiques nationales de suppression des subventions et/ou de privatisation des opérateurs occasionnent de fortes augmentations des tarifs électriques, les acteurs locaux peuvent encourager la maîtrise et la sobriété des consommations pour tenter de limiter les effets sociaux de ces hausses tarifaires, comme on le constate à Delhi. Pour lutter contre la pollution atmosphérique en ville, le charbon peut être remplacé par des énergies renouvelables, mais aussi par le gaz comme à Istanbul. Le coût d'extension des réseaux conventionnels dans les périphéries urbaines pourrait aussi favoriser les dispositifs décentralisés de production d'énergies renouvelables, même si aucun projet d'ampleur de ce type n'a été constaté. Ces contributions sélectives au changement énergétique, en fonction de mobiles socio-économiques plus qu'environnementaux, ne doivent toutefois pas masquer de nombreuses contradictions, qu'aggravent en outre les effets mal maîtrisés des politiques publiques de subvention et le clientélisme politique ${ }^{7}$.

À défaut d'être des lieux de pilotage du changement énergétique, les villes sont donc des sites stratégiques de déploiement des changements et de manifestation de leurs conséquences socio-politiques. L'approche comparée du changement a ainsi mis en lumière une politisation croissante de la question énergétique dans et par les villes. Exposées aux impatiences sociales et aux tensions qui résultent des changements en cours, les autorités urbaines contribuent à l'émergence d'un «problème public » (Hassenteufel, 2010), indissociable des dynamiques de résistance/négociation/contestation que manifestent les sociétés urbaines envers les formes contemporaines de la transition énergétique. Pour y faire face, elles déploient de nouveaux outils d'action publique (efficacité énergétique pour réduire leur dépenses propres mais aussi pour encadrer les usages et pratiques des consommateurs urbains face aux augmentations de tarifs). Elles se positionnent aussi comme interlocutrices des acteurs nationaux en accompagnant des revendications citadines (tarifs) ou en portant des revendications d'évolution du cadre réglementaire (pour permettre la production décentralisée d'énergie, le rachat d'énergies vertes...).

Les formes particulières que prend cette politisation sont très dépendantes des orga-

${ }^{7}$ Voir les études de cas dans : Jaglin et Verdeil (coords), 2013, Flux n 93/94. 
nisations et cultures politiques nationales, influencées par la nature des relations entre gouvernement métropolitain et État central et très marquées par les configurations énergétiques de chaque pays. Les contingences locales pèsent donc considérablement, ce dont l'approche comparative peut plus difficilement rendre compte.

\subsection{Focus sur un conflit : secteur électrique national et transversalité énergétique urbaine au Cap}

Les villes étudiées dans le projet Termos ne présentaient, a priori, aucun caractère d'exemplarité, Le Cap pas plus que les autres : elles ont été choisies pour des raisons contingentes liées aux parcours de recherche antérieurs des membres du projet, à leur capacité à mobiliser des connaissances contextualisées et des ressources pour répondre à la question commune sur le changement énergétique local ${ }^{8}$. Néanmoins, la comparaison a permis de définir des « profils territoriaux ». Celui du Cap, qui pourrait être qualifié d' « écologique audacieux mais contrarié », illustre une concurrence entre deux visions politiques de la transition énergétique : fondées sur l'introduction d'énergies renouvelables dans un mix électrique national jusqu'alors dominé par le charbon, elles s'opposent sur le poids respectif des logiques industrielles centralisées et des formes d'autonomisation locale dans l'organisation des systèmes électriques.

L'urbanisation des questions énergétiques au Cap est d'abord une urbanisation de la question électrique, dans un contexte qui reste surdéterminé par un cadre et des acteurs nationaux : le président et son cabinet, le ministère de l'Énergie (DoE), le régulateur national de l'énergie NERSA, l'entreprise nationale électrique ESKOM et son ministère de tutelle (le Département des Entreprises Publiques). Dans cette configuration, il s'agit d'analyser les actions de la municipalité dans le domaine électrique (en 2012, les plus gros consommateurs d'électricité sont les secteurs commercial (44\%) et résidentiel $(37 \%)$ et l'électricité représente $29 \%$ des consommations énergétiques totales de la métropole ${ }^{9}$ ) et leurs relations avec celles de la sphère centrale (Jaglin, 2014).

Pour décrypter la situation capetonienne, nous nous référons au travail d'Evrard, qui analyse le développement des énergies renouvelables en Europe comme un enjeu conflictuel opposant « un secteur et une alternative de politique publique » (Evrard, 2014 : 69). Le secteur est ici celui de l'électricité : en tant que sous-système d'action publique, il est doté d'une structuration dont l'auteur distingue trois principales dimensions : une dimen-

\footnotetext{
${ }^{8} \mathrm{Au}$ Cap, le travail de recherche est issu de plusieurs enquêtes de terrain menées entre 2010 et 2013 et reposant sur une vingtaine d'entretiens semi-directifs avec des acteurs locaux de la municipalité et du secteur électrique. Ces enquêtes ont été complétées par un travail de bibliographie et de collecte de sources primaires diversifiées : rapports d'étude, expertises techniques, comptes rendus de réunion du conseil municipal, presse locale et nationale accessibles à distance sur les sites internet de la municipalité, documents issus d'acteurs de la politique nationale énergétique (Eskom, gouvernement sud-africain, ministère de l'Énergie, ministère des Entreprises publiques), de centres d'expertise académiques (Energy Research Centre, University of Cape Town) ou encore d'agences et d'associations intervenant dans les énergies renouvelables (Sustainable Energy Africa, Greenpeace, Electricity Governance Initiative).

${ }^{9}$ Les carburants (essence et diesel) utilisés dans les transports urbains représentent $53 \%$ des consommations énergétiques totales (CoCT, 2015).
} 
sion sociale (un ensemble d'acteurs aux intérêts spécifiques); une dimension institutionnelle (des organisations formalisant les relations et les configurations de pouvoir); une dimension cognitive et normative (la vision dominante des problèmes et des solutions orientant les actions) (id. : 70). Dans de nombreux pays, le secteur électrique peut être ainsi décrit comme relevant d'une organisation centralisée fondée sur un modèle d'intégration verticale et des relations très étroites entre une ou un petit nombre de compagnies électriques et l'État. À cette organisation correspond un cercle étroit d'acteurs dominants, tous nationaux, détenant le quasi-monopole de l'élaboration des politiques énergétiques. Enfin, ces acteurs partagent une approche commune des questions énergétiques reposant sur la primauté des principes d'économie d'échelle, des logiques d'offre, des technologies de production de grande taille et des modes d'organisation centralisée.

En contrepoint, les énergies renouvelables ont été promues par des mouvements écologistes et antinucléaires, ainsi que certains experts, comme une voie alternative dotée d'une « ambition réformatrice » de l'ensemble du système énergétique voire de la société (Evrard, 2013). Définies comme « une alternative de politique publique », soit « un ensemble cohérent de propositions, construit autour de représentations communes, d'arrangements institutionnels et de configurations d'acteurs spécifiques, qui visent non seulement à promouvoir une solution particulière, mais également à transformer le secteur dans lequel celle-ci s'insère » (Evrard, 2014 : 73), les énergies renouvelables reposent en effet sur des représentations et des propositions susceptibles de subvertir le modèle hérité. Leur cadre cognitif emprunte à la notion de soft energy path (Lovins cité par Evrard : 73) et valorise les critères environnementaux, la sobriété et la maîtrise de la demande; le modèle institutionnel envisagé est fondé sur le développement d'une production dispersée et d'une gestion décentralisée; la gouvernance de ce nouveau secteur relève enfin d'acteurs plus nombreux, plus divers et aux rôles de producteur/consommateur davantage chevauchants.

Ce cadre d'analyse doit toutefois être adapté à notre démarche, centrée sur l'échelle et les acteurs métropolitains d'une part, l'ensemble des facteurs et modalités de changement de l'offre énergétique, d'autre part. Au conflit entre un secteur (de l'électricité) et une «alternative de politique publique » (les énergies renouvelables), il faut ainsi substituer une configuration dans laquelle le secteur national dominant de l'électricité est contesté par un «bloc» alternatif animé par des acteurs non sectoriels hétérogènes (la municipalité, des ONG, des experts/chercheurs, des usagers et des PME des énergies renouvelables). Organisé horizontalement à l'échelle métropolitaine, ce bloc est porteur de visions, représentations, approches des problèmes souvent décalés voire antagoniques de celles des acteurs dominants. Cette opposition s'est par exemple manifestée lors de la COP17 organisée à Durban en 2011 (SALGA \& SACN, 2011).

À la manière de Christen et Hamman (2015), on peut analyser les processus de changement au Cap comme résultant d'interactions entre une logique de transition-adaptation et une logique de transition-rupture. La première est portée par les acteurs historiques du secteur électrique qui s'emparent des innovations technico-scientifiques pour intégrer la demande de modernisation écologique au système existant; la seconde relève d'initiatives, plus dispersées et parfois concurrentes entre elles (Baker, 2011), pour lesquelles le changement passe par la resubstantialisation d'un projet collectif et la redéfinition locale de compromis pratiques dans le cadre de territoires relativement autonomisés du système national.

La figure dominante du conflit est ainsi entre un secteur électrique national et une 
approche alternative des questions énergétiques portée par des acteurs urbains et formulée à partir de leurs préoccupations, qui ne constitue pas un ensemble cohérent de propositions pour une transition énergétique nationale mais y introduit deux dimensions jusque-là étrangères au secteur électrique et potentiellement conflictuelles avec ses représentations et approches dominantes : la transversalité (complémentarité des sources d'énergie, maîtrise de la demande, désectorialisation intégrant énergie/transport/planification/bâtiment, etc.); la territorialisation (notamment par décentralisation d'une partie de la production mais aussi du contrôle et de la régulation au profit des acteurs locaux). Dans la littérature, la transversalité est justifiée par un nécessaire changement de paradigme, d'une offre toujours croissante vers un modèle plus sobre en consommation de ressources et fondé sur la maîtrise de la demande, l'efficacité des usages, le réemploi et les symbioses locales (Coutard, 2010). La territorialisation, au sens de relocalisation du contrôle sur la chaîne énergétique (de la production à la consommation), est diversement argumentée : comme un moyen, pour les villes, de sécuriser leur accès à des ressources énergétiques stratégiques, nécessaires à la poursuite de leur compétitivité économique (Hodson et Marvin, 2010); ou comme une ambition écologique de reconnecter gestion des ressources et développement local par « des arrangements pensés et négociés dans la proximité » (Durand et al., 2015 : 34). Ces divers arguments sont également mobilisés au Cap (Ward ${ }^{10}$ et Walsh, 2010) par un pouvoir municipal en quête de marges de manœuvre, comme en témoigne l'extrait d'un courrier de la maire Patricia de Lille (janvier 2015) : « To be responsible, we have to explore new energy options for local government specifically [...] We have to assess all of the options out there - from renewables to gas - and find the right sustainable combination for our current and future needs $»^{11}$.

\section{Le Cap : une marge de manœuvre étriquée}

Alors que Le Cap est identifié, tant en Afrique du Sud qu'à l'étranger (Simon et Leck, 2015), comme un champion de la transition écologique et énergétique et qu'un ensemble significatif d'actions y sont identifiables, le pouvoir municipal de transformation des politiques énergétiques semble faible. Certes, son interventionnisme vert a contribué, avec l'action d'autres acteurs (notamment des gouvernements métropolitains comme celui de Durban ${ }^{12}$ et des centres d'expertise environnementalistes comme Sustainable Energy Africa) à susciter des changements mais les logiques fondamentales du secteur électrique, principal bloc de résistance, n'ont pas, jusqu'à présent, été remises en cause. Dans une certaine mesure, ce secteur a même fait preuve de remarquables capacités d'adaptation, en intégrant certaines propositions alternatives, en adoptant leurs pratiques discursives et leurs récits, tout en conservant le pouvoir de contrôle.

\footnotetext{
${ }^{10}$ Sarah Ward est directrice du Energy \& Climate Change Environmental Resource Management Department du CoCT.

${ }^{11}$ En ligne sur le blog Cape Town Green Map : http://www.capetowngreenmap.co.za/blog/new-energysolutions-needed-cape-town (2015-01-30).

12 Voir Roberts (2010) et Taylor, Cartwright, Sutherland (2014).
} 


\subsection{Le secteur national : une puissante industrie électrique fragilisée}

Historiquement fondé sur l'utilisation d'un charbon à bas coût ( $85 \%$ de la production d'électricité), le secteur électrique sud-africain a jusqu'à la fin des années 2000 fournit une énergie abondante et très bon marché, favorable au développement d'une industrie minière puissante et, plus généralement, d'une production industrielle très intensive en énergie. L'acteur central de ce secteur est l'entreprise publique en monopole Eskom Holdings SOC Ltd, verticalement intégrée, détenue à $100 \%$ par l'État sud-africain (Jaglin et Dubresson, 2015a). Elle fournit $95 \%$ de l'électricité consommée en Afrique du Sud, possède la seule centrale nucléaire du continent et gère le plus grand système technique électrique africain. Au sein du système d'accumulation national progressivement construit en Afrique du Sud à partir de la fin du XIX ${ }^{\mathrm{e}}$ siècle, généralement qualifié de "Minerals-energy complex" ou MEC (Fine et Rustomjee 1996) et, plus récemment, de MEC+ (MacDonald 2009), les intérêts de l'opérateur électrique national sont organiquement liés à ceux des secteurs miniers et industriels et profondément marqués par les logiques de la rente extractive qui, loin de s'effacer, est au fondement d'une actuelle « reprimarisation » économique (Southall, 2010).

La production électrique issue des centrales thermiques à charbon, et dans une moindre mesure des centrales hydrauliques et nucléaire, est acheminée vers les villes par un réseau dont l'opérateur national est propriétaire et gestionnaire. Elle est ensuite distribuée conjointement par Eskom (dans certains quartiers et les zones d'activité industrielle) et par les municipalités qui disposent pour ce faire de leur propre réseau. Au Cap, la municipalité assure $75 \%$ de la distribution (en nombre d'abonnés) et l'entreprise nationale les $25 \%$ restants. Cette énergie électrique représente l'essentiel de l'énergie consommée par les usages résidentiels et commerciaux ${ }^{13}$.

Ce modèle électrique atteint aujourd'hui ses limites. À une période de surcapacité du parc de production dans les années 1980, a succédé une période de sous-investissement qui s'est traduite, en 2008, par une grave crise de l'approvisionnement et de nombreux délestages. Depuis lors, les dysfonctionnements du réseau s'accumulent, les états d'alerte sont périodiques, les coupures tournantes ont repris en 2014 et l'équilibre précaire entre capacité réelle de production et demande n'a été atténué qu'à la mi-2016. À cette crise électrique s'ajoute en outre le mauvais bilan sud-africain en termes climatiques, le pays étant, du fait du poids du charbon dans son économie, le douzième émetteur de gaz à effets de serre en 2013 (SEA, 2015). Pour y remédier, l'Afrique du Sud a adhéré aux objectifs du Protocole de Kyoto (2002) puis, en décembre 2009, le président Jacob Zuma s'est engagé, dans le cadre des Accords de Copenhague, à réduire les émissions de dioxyde de carbone de $34 \%$ en 2020 et de $42 \%$ d'ici $2025^{14}$.

La crise électrique en 2008 et une active diplomatie climatique ont ainsi conduit à l'affirmation d'une volonté politique nationale de transformer le paysage énergétique. Outre un grand programme d'investissements en partie financé par de spectaculaires augmentations du tarif moyen du kWh revendu $\left(0,20\right.$ rand $^{15}$ en $2007,0,61$ en $2012,0,89$ pro-

\footnotetext{
${ }^{13}$ Le pétrole lampant et le gaz liquide étant aussi utilisés par les ménages, notamment les plus pauvres.

${ }^{14} \mathrm{http}: / /$ www.thepresidency.gov.za/president/pr/2009/pr12061648.htm

${ }^{15}$ En $2015: 1$ Rand = 0,08 euro.
} 
grammé en 2017, soit une hausse de $345 \%$ (valeur courante) en dix ans), la crise a été un déclencheur dans la définition progressive d'une transition énergétique mettant ainsi fin à « une décennie perdue » (les années 2000) du point de vue de la modernisation du système électrique (Gaunt 2008) ${ }^{16}$ comme de la réflexion sur les énergies renouvelables (Krupa et Burch 2011; Pegels 2009). Parmi les initiatives, mentionnons l'adoption de l'Integrated Resource Plan (IRP) 2010-2030, le Green Economy Accord ${ }^{17}$, l'incorporation d'objectifs « verts » dans l'Industrial Action Plan (IPAP2), et surtout, le lancement du Renewable Energy Independent Power Producer Procurement Programme (REIPPPP). Avec ces nouvelles procédures, le gouvernement a enfin les moyens de concilier les enjeux énergétiques et les enjeux de développement, les industries vertes étant désormais considérées comme un levier de l'emploi dans l'industrie manufacturière.

Jusqu'alors, la diversification du bouquet énergétique n'avait été que très modestement affirmée au profit de mesures pour la plupart sociales, comme l'accès à l'électricité pour tous, dans le Livre blanc sur l'énergie de 1998. Si le Livre blanc sur les énergies renouvelables (novembre 2003) puis l'approbation en septembre 2005 du Renewable Energy Subsidy Scheme par le ministère de l'Énergie (DoE) et le Trésor National avaient ensuite posé les bases d'un nouveau cadre, les lenteurs de la transition énergétique en Afrique du Sud avaient été soulignées lors du Sommet sur les énergies renouvelables, tenu en 2009 à Pretoria. Il faut donc attendre le début des années 2010 pour observer une reprise en main plus lisible de la transformation du cadre d'action, quand le DoE rend public l'IRP, un plan à 20 ans pour le secteur électrique. Ce plan évalue la demande électrique jusqu'en 2030, détermine le mix technologique et le bouquet énergétique, le partage entre Eskom et d'autres producteurs indépendants, les plans de financement à court, moyen et long termes et il fixe la trajectoire des tarifs. Il résulte de propositions d'un comité technique créé en février 2010, constitué de protagonistes principalement issus du DoE, d'Eskom et du Energy Intensive Users Group (EIUG) ${ }^{18}$, la System Operations and Planning Division d'Eskom assurant les modélisations. En cela, il reflète avant tout les intérêts des acteurs nationaux du secteur électrique et de ceux qui en dépendent étroitement.

Le secteur national de l'électricité apparaît ainsi très unifié autour d'Eskom et d'un groupe d'acteurs cimentés par une même vision du rôle historique de l'entreprise nationale : fournir de l'énergie électrique au plus bas coût possible, favoriser la reproduction du complexe minéralo-énergétique (Fine et Rustomjee, 1996), moteur historique de l'économie sud-africaine, permettre une diversification industrielle. Du fait de son rôle central dans la construction et l'évolution du complexe politico-économique sud-africain,

\footnotetext{
${ }^{16}$ Ces réformes du secteur électrique, inspirées de modèles orthodoxes - privatisation, libéralisation, régionalisation -, ont cristallisé des conflits d'intérêts qui en ont bloqué la mise en œuvre. À partir de 2010-2011, les réformes les plus contestées sont abandonnées - notamment la régionalisation de la distribution électrique, contre laquelle se sont élevés les gouvernements métropolitains (Gaunt 2008; Jaglin 2014) - et un cadre plus stable est progressivement mis en œuvre.

${ }^{17}$ À travers cet accord, le gouvernement s'est engagé à installer 3725 MW d'énergie renouvelable en 2016 et à créer 50,000 emplois verts en 2020 dans les industries du solaire et de l'éolien (South African Government Information : http://www.info.gov.za/speech/DynamicAction?pageid=461\&tid=50584).

${ }^{18}$ L'EIUG represente 36 firmes importantes qui, ensemble, consomment environ $40 \%$ de l'électricité vendue en Afrique du Sud (Idasa et al., 2010).
} 
le secteur électrique ne se comprend pas hors des relations organiques entre la construction d'Eskom et celle du pouvoir d'État, avant, pendant et après l'apartheid (Jaglin et Dubresson, 2015a). Or, cette articulation est aujourd'hui source de fragilité : face à la crise électrique et aux implications des engagements climatiques pris par le président Zuma, le secteur électrique, et notamment Eskom, est a priori mal armé pour sortir de l'addiction du charbon et piloter la transition énergétique. En effet, les compétences technologiques dominantes sont liées aux énergies fossiles, le système d'innovation national est fortement biaisé en faveur de ces mêmes énergies (Baker, 2011; Pegels, 2009) et l'intégration des énergies renouvelables, non souhaitée par Eskom, a été conflictuelle (Morris et Martin, 2015). Plus généralement, les acteurs de la sphère centrale semblent surtout soucieux de contrer les approches énergétiques alternatives qui menacent l'exclusivité de leur contrôle sur le secteur électrique.

\subsection{Au Cap, un volontarisme vert affiché19}

Avec l'IRP et le REIPPPP, une nouvelle donne pour les énergies renouvelables a été élaborée à l'échelle nationale, accompagnant un changement des modes d'action de la sphère centrale et suscitant, en retour, un ajustement aux échelles métropolitaines. Des agendas verts municipaux existaient avant la crise électrique et l'IRP, mais ces derniers en ont rendu urgente l'application. Au Cap, deuxième ville du pays avec 4 millions d'habitants en 2016, où les premières initiatives vertes datent de 1998, une stratégie de transition a été mise au point durant la décennie 2000 et, en 2010, un plan d'action visant à porter la part des énergies renouvelables à $10 \%$ du mix énergétique local en 2020, puis à environ 1/3 en 2050, a été approuvé. La municipalité s'est donc rapidement et activement mobilisée autour des questions énergétiques et de climat et son activisme vert bénéficie d'une image positive (Cartwright et al. 2012; Jaglin 2014; Mans 2010).

Ce dernier ne va cependant pas de soi dans un contexte où les spectaculaires hausses des tarifs et les délestages électriques ont des conséquences directes à l'échelle des villes et des métropoles ${ }^{20}$ (SEA, 2015). L'électricité est en effet une ressource stratégique pour les gouvernements urbains locaux ${ }^{21}$. D'une part, achetée en gros à Eskom puis revendue, elle produit des excédents mobilisés pour financer des péréquations internes ainsi que des mesures sociales en faveur des citadins pauvres. D'autre part, définies comme des gouvernements de développement local dans le Municipal System Act, $n^{\circ} 32,2000$, ces municipalités ont une obligation de soutien au développement économique et les tarifs de l'électricité comme la régularité et la qualité du service sont des facteurs essentiels de production et de compétitivité. Sortir du sentier de dépendance imposé et défendu par le

\footnotetext{
${ }^{19}$ Cette sous-partie reprend des extraits d'une communication présentée avec Alain Dubresson lors du séminaire final du projet Termos («Municipalities, major actors in energy change? Lessons from Cape Town », communication for the Urban energy governances, North and South International roundtable seminar, Gif-surYvette, Paris, 16-18 September 2013).

${ }^{20}$ Le Cap fait partie des 8 municipalités métropolitaines sud-africaines, qui appartiennent à la «sphère locale » de pouvoir dans le système de gouvernement multiniveaux.

${ }^{21} \mathrm{Au} 30$ juin 2015, la vente d'électricité est la deuxième source de revenus des municipalités (28,3\% soit R86,4 milliards) après les dotations et subventions (31\%) (Statistics South Africa 2015).
} 
secteur électrique national exige donc la construction d'une véritable « infrastructure » au service d'une approche énergétique urbaine alternative.

\subsubsection{La formation d'une coalition métropolitaine verte}

Les enjeux de la transition énergétique ont en effet mobilisé efficacement des acteurs de tous types, publics, privés marchands et associatifs, qui sont parvenus à créer un dispositif commun de réflexion et de proposition à l'issue d'une décennie d'intense production de rapports d'expertise et de documents prospectifs. On peut y voir une expression de spécificités du Cap. La quête d'un relatif affranchissement de la dépendance du réseau national, dont la gestion trop centralisée et bureaucratique entraverait les innovations locales, est une constante du discours tenu par les grandes entreprises privées et la municipalité (Jaglin, 2014). Quant aux nombreuses et très actives associations militantes vertes, elles prônent depuis fort longtemps un autre chemin de croissance. L'accent est ainsi mis sur la qualité environnementale de la péninsule, brandie comme une arme de marketing international pour magnifier le Cape lifestyle, capter des IDE et faire progresser rapidement la ville dans tous les classements internationaux. Le nécessaire changement d'une image qui surévalue la rente touristique et l'immobilier de haut de gamme pour riches retraités, la quête d'une rupture avec le business as usual et la promotion d'une ville entrepreneuriale, verte et durable nourrissent un discours décarboné qui valorise les énergies renouvelables, solaire et éolienne en particulier, également présentées comme d'efficaces outils de lutte contre la pauvreté et la précarité énergétique.

De fait, les conditions climatiques locales sont favorables. Le Cap reçoit $2198 \mathrm{kWh} /$ $\mathrm{m}^{2} /$ an de rayonnement solaire et, sur un capteur à haute performance, il est possible d'obtenir en moyenne $6,02 \mathrm{kWh} / \mathrm{m}^{2} /$ jour, soit $15 \%$ de plus que la moyenne nationale. Quant au potentiel éolien, c'est l'un des plus élevés d'Afrique du Sud selon le Wind Atlas of South Africa ${ }^{22}$.

L'intérêt de ces données n'a pas échappé aux activistes verts et à la municipalité, ni non plus au gouvernement provincial. En 2000, le Western Cape, qui produisait alors $26 \%$ de la valeur ajoutée des énergies renouvelables nationales, était déjà considéré comme un haut lieu d'expertise et en 2012, le ministre provincial de l'Economic Development and Tourism, déclarait que le Western Cape devait devenir le hub vert de l'Afrique du $\mathrm{Sud}^{23}$.

C'est donc dans un contexte favorable qu'a émergé la coalition métropolitaine verte du Cap, née d'une initiative de la municipalité. Le 22 septembre 2009, lors de la première réunion du Climate Change Think Tank, financé par l'ambassade du Danemark, 30 universitaires et militants verts se sont engagés à dresser un état des lieux et à proposer une évaluation prospective des conséquences du changement climatique au Cap. Le travail du groupe, synthétisé dans un ouvrage (Cartwright et. al. 2012), vise à guider la définition et la mise en œuvre d'une politique publique de l'énergie et du climat. Le moment décisif est issu de la candidature du Cap à l'accueil de la COP 17, finalement organisée à Durban fin 2011, et de l'élaboration de propositions communes à tous les acteurs impliqués, fédérant des initiatives auparavant dispersées. Le 2 mars 2012, lors de l'ICLEI Conference on Local Climate Solution for Africa 2011 (Le Cap, 27 avril-3 mars), a été créée la Cape

\footnotetext{
${ }^{22}$ http://www.wasa.csir.co.za.

${ }^{23}$ « Winde sees Western Cape green economy jobs potential », Engineering News, 10 August 2012.
} 
Town Climate Change Coalition, regroupant des acteurs publics, privés marchands et associatifs. Cet activisme militant est concomitant, d'une part, de l'intégration de la question énergétique dans les priorités municipales, d'autre part, d'ajustements institutionnels internes à la municipalité.

\subsubsection{L'expression du volontarisme : l'ajustement institutionnel et la planification}

«verte»

L'énergie, vecteur de la ville durable, a été ajoutée à la liste des objectifs stratégiques du plan intégré de développement (IDP) du Cap en 2008. La même année a été créé l'Energy and Climate Change Committee, qui facilite la coopération entre le Mayoral Committee et l'administration, les deux grandes composantes politique et technique du dispositif municipal. Pour susciter les échanges entre les départements administratifs concernés d'une part, entre ces départements et les conseillers élus d'autre part, trois groupes de travail transversaux ont été mis en place : Energy Security and Carbon Mitigation; Adaptation and Climat Resilience ; Communication and Education. Au sein de l'administration municipale, l'approche énergie durable est coordonnée et promue par le City's Environmental Resource Management Department, en charge des projets et des programmes, tandis que la réflexion et l'élaboration des documents sont confiées à l'Energy and Climate Change Unit, dans laquelle ont été recrutés des militants verts très actifs.

La crise de l'approvisionnement en électricité en 2008 ayant accéléré la mobilisation de la municipalité, l'Energy and Climate Change Strategy de 2006 donne naissance à un plan plus ambitieux approuvé par le conseil municipal en mai 2010 : l'Energy and Climate Action Plan (ECAP). Ce plan global et intégré propose 11 objectifs complémentaires visant à réduire la consommation énergétique urbaine, à promouvoir les énergies renouvelables, censées fournir $10 \%$ de l'approvisionnement énergétique du Cap dès 2020, et à stimuler le développement économique local via ces énergies (CoCT, 2011). Riche en données et indicateurs, le rapport Cape Town State of Energy (2011 puis 2015) présente pour sa part le bilan actualisé de la politique municipale, dont il constitue un tableau de bord accessible à tous (CoCT, 2015).

L'ECAP décline deux grands types d'action. Les unes visent à promouvoir des économies et des usages plus efficaces de l'énergie : c'est actuellement le domaine d'intervention le plus significatif à l'échelle locale. Les autres visent le soutien à la production d'énergie renouvelable et la structuration d'une économie verte locale : dans ce domaine toutefois, le champ d'action est encore limité.

Les initiatives en matière d'efficacité énergétique ciblent les bâtiments et véhicules de la municipalité ainsi que les secteurs résidentiels, commerciaux et industriels privés dans lesquels le $\mathrm{CoCT}$ est en mesure de jouer un rôle de facilitateur. Ces interventions sont encore souvent expérimentales, dans l'attente d'une méthodologie adaptée de mesure des rapports coût-efficacité de différentes options et de données plus précises, à l'échelle de la métropole, sur les habitudes de consommation d'énergie et les durées d'amortissement de divers dispositifs d'économie d'énergie. Afin de fournir aux propriétaires et gestionnaires de bureaux, de centres commerciaux, d'hôtels et d'autres bâtiments commerciaux et publics une connaissance pratique des solutions d'efficacité énergétique disponible et de proposer une plate-forme de mutualisation pour l'action collective et l'apprentissage 
partagé ${ }^{24}$, le CoCT a mis en place un Energy Efficient Forum. Des réunions régulières permettent des échanges autour d'études de cas en situation réelle, des discussions et des mises à jour sur les options de financement et les questions posées par les politiques publiques. Le Cape Town Partnership ${ }^{25}$, qui soutient cette initiative, et l'Institut de développement durable (lié à l'Université de Stellenbosch) ont également joué un rôle actif dans la promotion de l'efficacité énergétique des immeubles de bureau du CBD, tandis que le centre d'expertise Sustainable Energy Africa (SEA) a développé, avec un financement de l'organisation de développement néerlandaise Hivos, une nouvelle ressource en ligne pour améliorer la connaissance des entreprises de services énergétiques (ESCO) basées au Cap, montrant ainsi qu'une économie locale de l'efficacité énergétique et de la gestion de la demande est en train d'émerger.

Ces efforts produisent des résultats en termes de marketing vert. Ainsi, dans le CBD, l'opération immobilière Portside, d'un coût de R1,6 milliards, dans laquelle le plus haut bâtiment du Cap a été érigé (34 niveaux et $69000 \mathrm{~m}^{2}$ de surface de plancher brute combinée), a obtenu cinq étoiles du Green Building Council de l'Afrique du Sud (Engineering News online, $19^{\text {th }}$ July 2013), tandis que l'opération Silo ${ }^{\circ} 1$, immeuble de bureaux (9 étages) situé au Victoria \& Alfred Waterfront, inauguré en 2014, est le deuxième bâtiment du pays a obtenir une cote de six étoiles, le premier dans le Western Cape (Engineering News online, 22 ${ }^{\text {nd }}$ February 2013). Dans un domaine à l'interface des économies d'énergie et des énergies vertes, le Solar Water Heater Advancement Programme (SWHAP) est aussi un bon exemple des ambitions et des limites de l'action municipale (Dubresson, 2013). Celle-ci a franchi une nouvelle étape avec la signature, le 23 septembre 2014, d'un contrat d'achat du surplus d'électricité produit par les $11000 \mathrm{~m}^{2}$ de panneaux photovoltaïques posés sur les toits du parc d'affaires Black River Park (1,2 MW installés, 1368 MWh produits) localisé à Observatory. Depuis, un modèle de partenariat favorisant les petits producteurs électriques autonomes a été mis en place.

L'approche alternative des questions énergétiques que propose Le CoCT passe donc par une présence active dans les organisations et réseaux internationaux, des discours politiques fermes et constants en faveur de la transition énergétique, un ajustement institutionnel interne qui s'appuie sur la production d'expertise et de plans ainsi que sur la construction d'instruments d'action publique nouveaux. L'émergence d'une coalition verte composée d'acteurs qui ne sont pas des acteurs traditionnels du secteur électrique et l'approche désectorialisée des questions énergétiques ${ }^{26}$, associant offres alternatives de sources diverses, efficacité énergétique et maîtrise de la consommation, couplée à des politiques centrées sur des problèmes urbains locaux définissent une véritable proposition alternative aux solutions prônées par le secteur électrique national. Cependant, si l'on observe le déploiement métropolitain de nombreuses actions, cette incontestable

\footnotetext{
${ }^{24}$ Site officiel du CoCT : http://www.capetown.gov.za/en/EnergyForum/Pages/default.aspx

${ }^{25}$ Le Cape Town Partnership est un partenariat public-privé en charge du développement et de la promotion du centre-ville.

${ }^{26}$ Il faut ajouter que le CoCT est aussi actif dans le domaine des transports : le lancement, en mai 2011, de la première phase d'un nouveau système de transport intégré par autobus, MyCiti, illustre la volonté municipale de réduire à la fois la congestion et la pollution liées à l'intense circulation automobile individuelle (le déploiement du réseau en quatre phases doit durer 25 ans).
} 
urbanisation des questions électriques ne conduit pas à leur territorialisation et ne favorise pas une décentralisation positionnant le CoCT comme un acteur important du système électrique. Au Cap comme ailleurs existent des problèmes de capacité et d'efficacité de l'action urbaine locale. La construction d'une gouvernance horizontale est difficile, comme le montre la laborieuse coopération entre des services municipaux aux cultures professionnelles différentes dans les efforts de mise en œuvre du SWHAP (Froestad et al. 2012). Ces dysfonctionnements locaux sont de surcroît amplifiés par des facteurs de résistance ancrés au cœur du secteur national de l'électricité.

\section{Blocages et hybridations : une territorialisation freinée par le secteur national de l'électricité}

En dépit du volontarisme public affiché, les actions réalisées par le CoCT demeurent, à ce stade, peu nombreuses : concurrencées par un nouveau programme national, les ambitions du SWHAP ont été réduites, le projet éolien de Darling Farm est un demi-échec, le reste de la production verte, piloté via le REIPPPP, échappe totalement à la municipalité. Les transformations du système électrique, tout en favorisant les producteurs privés et les énergies renouvelables, sont donc pour l'heure insuffisantes pour promouvoir sa territorialisation métropolitaine et une transformation significative du modèle énergétique dominant : même défaillant, Eskom, reste aux commandes; les solutions à la crise demeurent surdéterminées par les représentations dominantes des acteurs du secteur électrique et minier (Jaglin, Dubresson, 2015b); la centralisation des organisations et de la gouvernance n'est pas ébranlée. Cette résilience peut s'expliquer de deux manières complémentaires : la résistance de la sphère centrale à des évolutions qui nécessitent une redistribution ou un partage du pouvoir de contrôle sur le secteur électrique; la capacité de cette même sphère centrale à intégrer des propositions qui assurent une adaptation du secteur sans menacer son pouvoir.

\subsection{Incertitudes institutionnelles et concurrences : des freins à l'action publique locale}

Si des évolutions du système énergétique sont en cours, la tentative de transformer radicalement la manière d'aborder les questions énergétiques aux échelles locales et la place qu'y occupent les logiques sectorielles de l'électricité s'est révélée, pour le moment, en grande partie infructueuse.

\subsubsection{Désajustements réglementaires}

En dépit d'initiatives techniques de qualité et d'innovations institutionnelles incontestables, les municipalités restent dépendantes, pour la mise en œuvre de nombreux programmes, des évolutions réglementaires nationales, lesquelles évoluent lentement et, du point de vue de nombreux acteurs en quête de perspectives stables, de manière erratique.

Ce fut le cas pour le SWHAP du Cap, longtemps freiné par les National Building Regulations (NBRs) et aujourd'hui contraint par elles. Les NBRs fixent les normes de construction des bâtiments, au respect desquelles doivent veiller les municipalités délivrant les permis de construire. En termes d'énergies renouvelables, leur modification était donc l'une des plus importantes et des plus attendues. Mis en discussion en juillet 2010, les 
amendements au National Building Regulations and Building Standards Act 1977 (Act 103 of 1977) ont été notifiés aux municipalités le 9 septembre 2011 pour une entrée en vigueur à partir du 10 novembre 2011. Ces amendements stipulent que les modalités de chauffage de l'eau et d'isolation d'un bâtiment doivent figurer dans la demande de permis de construire et surtout que, pour toute construction nouvelle ou extension de construction existante, $50 \%$ au moins de l'énergie utilisée doivent provenir de sources renouvelables. Ces normes ont des implications opérationnelles fortes : dans l'état des techniques et des coûts de production du kWh en 2011, les chauffe-eau solaires et les pompes à chaleur étaient alors les mieux à même de répondre à cet impératif, ce qui ne laissait guère d'autres options aux programmes municipaux, lesquels peuvent toutefois, en 2017, miser un peu plus sur le photovoltaïque, dont les coûts ont été considérablement réduits. En outre, le contrôle technique des bâtiments exige un personnel d'inspection dont le Planning and Building Development Management Department municipal ne dispose pas ou plus à l'issue d'une décennie de restructuration administrative (Froestad et.al.2012).

Darling Wind Farm est un bon exemple de frein à l'action municipale faute d'une politique nationale consolidée. Une infime partie de l'électricité consommée au Cap $(0,1 \%)$ est achetée à la ferme éolienne privée de Darling, premier parc éolien indépendant construit en Afrique du Sud et mis en service en mai 2008. Le CoCT a commencé à vendre des certificats verts (GECs) en mai 2010, avant qu'un cadre officiel ait été établi par le gouvernement pour réglementer ce marché, en développant un système propre de règles et de normes ${ }^{27}$. Même si ces certificats concernent principalement les entreprises et pour un très faible pourcentage de leur énergie, le CoCT prenait ainsi un risque, dénoncé par des experts nationaux pour qui les certificats verts municipaux n'étaient ni légaux ni commercialisables en dehors d'un cadre national reconnu. De fait, la ferme produit 6-8 GWh par an soit, depuis mai 2008, un total d'environ 30,8 GWh d'électricité verte, dont seulement 1,8 GWh ont été vendus par le CoCT sous forme de certificats verts. Le CoCT est lié à la ferme éolienne de Darling par un accord d'achat de 20 ans mais celuici ne sera ni prolongé ni renouvelé selon le responsable municipal concerné : "there is no great potential in the short term" 28 . En outre, le CoCT n'envisage aucune nouvelle expérience dans ce domaine tant que la loi nationale sur les finances locales (Municipal Finance Management Act) n'aura pas été adaptée aux exigences de ce type de contrat.

Ces exemples mettent en évidence les entraves aux initiatives locales liées aux lenteurs ou résistances d'adaptation du cadre juridique national en vigueur (Mans 2010). Le rapport 2015 State of Energy in South African Cities rend également compte de ce flou institutionnel (SEA, 2015).

\subsubsection{Concurrence et initiatives rivales}

Un autre frein au déploiement des alternatives locales est la concurrence qu'exercent les initiatives de la sphère centrale. Ainsi, les réponses à la crise électrique diffèrent : tandis que des membres de la coalition verte du Cap promeuvent la maîtrise de la demande, l'efficacité énergétique ou encore le déploiement du solaire thermique, Eskom insiste sur la nécessité de renforcer ses capacités de production nationales par de nouveaux pro-

\footnotetext{
${ }^{27}$ Entretien : Head of Green Energy, Municipal Electricity Services, août 2011.

${ }^{28} \mathrm{Id}$.
} 
jets de centrales pour satisfaire l'accroissement anticipé de la demande future. Ces priorités ont été réaffirmées dans l'IRP 2010 qui, en dépit d'un engagement ambitieux en faveur des énergies renouvelables, témoigne aussi du poids persistant des combustibles fossiles (charbon et hydrocarbures). Il met surtout l'accent sur deux priorités, accroître les capacités de production et diversifier les sources d'énergie, conduisant à la poursuite des investissements dans les technologies du charbon propre et du nucléaire. L'IRP est cependant, depuis sa publication en 2011, au centre de vives polémiques et ses scénarios de croissance sont très vivement contestés à la fois par nombre d'experts et de militants verts dénonçant l'influence des intérêts représentés dans le comité technique, notamment ceux de l'EIUG (Idasa et al. 2010; Pickering 2010). Ses objectifs divergent aussi des perspectives de changement défendues par les métropoles, à partir d'une approche locale et transversale des questions énergétiques, et pour lesquelles elles réclament plus de coordination et de soutien de la part du gouvernement national (SEA, 2015).

Même le modeste secteur d'activité des chauffe-eau solaire suscite une compétition contreproductive entre la municipalité du Cap et Eskom. En 2008, celle-ci a lancé le programme national Demand Side Management (DSM), doté d'un budget de 10 milliards de rands permettant de financer plusieurs actions, dont l'installation de 950000 chauffe-eau solaires en 2013 grâce à un mécanisme de subvention à l'achat et à l'installation. En 2010, pour stimuler la diffusion de chauffe-eau solaires, a été créé le National Solar Water Heater Programme (NSWHP) dont la dernière version vise l'installation de 5,6 millions de chauffe-eau solaires d'ici 2020 avec l'aide de subventions à la demande et dans le cadre de normes nationales qui ne prennent pas en compte les intérêts de la filière sud-africaine, dont la production est en concurrence sur le marché national avec les produits chinois importés. À l'inverse, le programme SWHAP du Cap tente de combiner soutien à la demande et soutien à l'offre en améliorant la qualité des relations entre les acteurs de la chaîne de valeur productive locale (fabricants, fournisseurs et banques) tout en cherchant à structurer l'industrie à l'échelle métropolitaine ou provinciale (Dubresson, 2013). Au-delà des difficultés de mise en œuvre du programme national (en janvier 2014, toutes opérations publiques et privées confondues, 395000 appareils seulement étaient posés), un constat s'impose : les municipalités métropolitaines ont été marginalisées dans les choix opérés comme dans les procédures adoptées par le NSWHP, tandis que les fluctuations permanentes du cadre normatif national contraignent voire contrecarrent leurs propres initiatives. Cet exemple illustre de manière significative la divergence entre une approche sectorielle, celle d'Eskom, et une approche transversale de développement local, celle de la municipalité, chacune ayant ses propres priorités et contraintes.

\subsection{Appropriations et hybridations : l'exemple du REIPPPP}

Les acteurs dominants de l'électricité ne sont cependant pas uniquement dans une stratégie d'opposition; leur force réside aussi dans leur capacité à intégrer une partie des propositions alternatives dans leurs propres discours, comme en témoignent les évolutions discursives insistant sur la croissance verte et le charbon propre (Jaglin et Dubresson, 2015b), et dans leurs objectifs : le Renewable Energy Independent Power Producer Procurement Programme (REIPPPP) en est une illustration.

Ainsi, tout en poursuivant leurs investissements dans les technologies du charbon 
propre et du nucléaire, le pouvoir d'État et certains départements d'Eskom se sont résignés, sous l'effet de la crise électrique de 2007-2008 et de ses conséquences en termes de hausses spectaculaires et répétées du tarif de base, à mettre en œuvre des mécanismes favorables aux énergies vertes et à une transition énergétique réduisant la dépendance aux sources d'énergie carbonées (Baker, 2011). L'enjeu étant national et stratégique, cette résignation est accompagnée d'une reprise en main par le ministère de l'Énergie, le Trésor national et le régulateur national NERSA qui limite la compétence directe d'Eskom et ignore les municipalités métropolitaines dans le pilotage du nouveau dispositif.

$\mathrm{Au}$ cœur de ce dernier se trouvent les tarifs de rachat aux producteurs privés et les modalités de sélection des projets, qui reposent sur un système d'appels d'offres concurrentiel. Le premier des cinq appels d'offres prévus a été mis en ligne par le ministère de l'Énergie le 3 août 2011 : les soumissionnaires sont appelés à répondre en proposant des prix de vente par MWh, pour les vingt premières années de mise en service ou pour la durée du contrat d'achat, en fonction de la technologie adoptée (le prix proposé compte pour $70 \%$ de la note attribuée dans les critères de sélection), et en détaillant les effets d'entraînement sur le développement local (30 \% de la note). Le ministère de l'Énergie invite les développeurs potentiels à soumettre des offres pour le financement, la construction, l'exploitation et l'entretien de diverses infrastructures et technologies : éolien terrestre, centrales solaires, solaire photovoltaïque, biomasse, biogaz, gaz de décharge ou petites centrales hydroélectriques. Les soumissionnaires sélectionnés par le ministère de l'Énergie sont ensuite signataires d'un accord de rachat avec Eskom (Eberhard et al., 2014). Le succès des quatre premiers appels d'offres est incontestable : en avril 2015, la capacité de production ${ }^{29}$ des 79 projets sélectionnés sur les 225 reçus atteint $5243 \mathrm{MW}$ pour un investissement total estimé à R168 milliards (DoE, 2015).

Cette réussite ne s'accompagne toutefois pas d'une décentralisation du système de gouvernance ou d'une approche plus intégrée des questions énergétiques et de développement urbain. Signataire des contrats d'achat aux producteurs privés, Eskom est partie prenante de la production d'énergies renouvelables, dont les règles nationales sont maîtrisées par le ministère de l'Énergie et le NERSA, appuyés par le Trésor National, qui ont mis en place des unités spéciales au sein de leur propre dispositif pour piloter efficacement tout le processus (Eberhard et al., 2014). Les gouvernements locaux, et notamment les métropoles, ne définissent ni ne contrôlent aucune décision stratégique : le total de la capacité attribuable, la répartition par types d'énergie, les procédures à suivre ou encore le contenu des $30 \%$ de la note finale d'évaluation des projets correspondant au développement économique induit. Les acteurs dominants ont ainsi repris une des demandes des promoteurs d'approches énergétiques alternatives en intégrant des sources renouvelables dans le secteur électrique tout en écartant les propositions les plus radicales : si la production est locale, l'amont (les appels d'offres) et l'aval (les conditions de rachat, de transport $^{30}$ et de distribution) restent contrôlés centralement.

\footnotetext{
${ }^{29}$ Cette capacité est pour le moment théorique car l'achèvement des projets sélectionnés est progressif (au 31 mars 2016, 43 étaient entrés en production commerciale).

${ }^{30}$ En avril 2015, la KfW allemande a ainsi accordé à Eskom un prêt de R4-billion (\$339 millions) pour renforcer son réseau et y connecter les nouvelles centrales éoliennes et solaires.
} 


\section{Conclusion}

L'épuisement structurel du système électrique sud-africain et, sur le court terme, la crise de 2008 ont entraîné des changements à la fois dans la politique nationale - inflexion notable en faveur des énergies renouvelables, ouverture aux producteurs privés - et dans le positionnement stratégique des métropoles, notamment du Cap, mais les modèles s'opposent. En dépit de leurs désaccords, les acteurs de la sphère centrale sont jusqu'à présent parvenus à conserver le contrôle du secteur électrique en y intégrant les énergies renouvelables délestées du projet alternatif dont sont porteurs nombre de leurs partisans. En conséquence, le paysage énergétique sud-africain change et incorpore une partie des propositions alternatives des acteurs urbains sans toutefois conférer à ces derniers une place accrue dans le secteur électrique. Ce contexte n'est pas favorable à la construction d'une autonomie énergétique locale ni à la décompression gestionnaire aux échelles infranationales. S'il n'empêche pas la mise en œuvre de programmes nationaux à l'échelle locale urbaine, il ne permet pas aux municipalités sud-africaines de devenir des acteurs majeurs de la production d'énergies renouvelables, d'autant que la Constitution de 1996 ne leur donne pas mandat de produire de l'électricité, fut-elle «verte».

Au-delà du Cap et de l'Afrique du Sud, trois enseignements de portée générale peuvent être formulés. Premièrement, avant d'être un enjeu du développement durable et de lutte contre le changement climatique, les politiques énergétiques sont, dans les pays émergents, d'abord des outils d'impulsion du développement économique et social; elles restent dominées par des enjeux industriels et techniques ainsi que par des coalitions politico-industrielles nationales. Deuxièmement, le «verdissement » des systèmes énergétiques n'est pas corrélé à la transformation institutionnelle de ces derniers. Ce constat est partagé dans d'autres contextes, par exemple à Los Angeles, où le système énergétique a été adapté pour intégrer une part significative d'énergies renouvelables sans remise en cause de son organisation (Monstadt et Wolf, 2015). Troisièmement, le rescaling ne se traduit pas de manière identique pour toutes les composantes des systèmes énergétiques, la déconcentration géographique de l'appareil de production qui accompagne le déploiement des énergies renouvelables pouvant s'accommoder de modes de contrôle très centralisés du système électrique.

Ce découplage des échelles (Bridge et al., 2013) se double en outre fréquemment d'une disjonction entre les dispositifs techniques et les visées réformatrices des alternatives énergétiques. En effet, les premiers peuvent être intégrés dans la gestion centralisée des acteurs du secteur électrique d'une manière qui les dissocie des forces de contestation de l'organisation historique du secteur électrique et des approches alternatives intégrées et transversales que défendent les acteurs urbains. C'est la situation que décrit Evrard en Europe, où les acteurs dominants du secteur électrique ont domestiqué les technologies de production des énergies renouvelables tout en marginalisant le projet de transformation sociale dont étaient porteurs certains de leurs partisans (Evrard, 2013). Plus nuancée, la conclusion de Christen et Hamman (2015) évoque plutôt, à partir du cas alsacien, des processus d'imbrication et d'hybridation des logiques industrielles centralisées et des initiatives citoyennes alternatives. Dans ce continuum, Le Cap révèle une configuration intermédiaire dans laquelle le secteur électrique national se transforme par hétérogénéisation relative de son système technique et incorporation partielle de certaines propositions des acteurs urbains. 
Ces découplages et disjonctions expliquent un apparent paradoxe, souligné par Termos : celui d'une urbanisation croissante des enjeux de la transition énergétique qui ne témoigne (pour le moment du moins) ni d'une remise en cause de l'organisation centralisée des secteurs électriques nationaux, ni d'une approche plus intégrée des questions énergétiques et de développement urbain, ni enfin d'une meilleure représentation des gouvernements locaux, notamment métropolitains, dans la gouvernance des systèmes énergétiques. Ils invitent surtout à discuter l'hypothèse de substitution associée, plus ou moins explicitement, à la notion de transition, car les observations de terrain donnent à voir une concomitance : des logiques industrielles et alternatives, des réseaux centralisés et des dispositifs dispersés, des énergies fossiles et renouvelables... Dans l'exemple sud-africain, cette concomitance est régulée par des acteurs nationaux qui suscitent/ sélectionnent/formalisent les initiatives locales puis encouragent leur appropriation par les acteurs industriels du secteur. Autrement dit, la sphère centrale perd le monopole de l'innovation, partagé avec la sphère locale urbaine et des acteurs « périphériques » (des petites entreprises des énergies renouvelables voire des communautés rurales) mais réaffirme son contrôle sur les conditions de mise en œuvre, les gagnants politiques de ce mouvement étant, au stade actuel, les acteurs de la sphère centrale de pouvoir. Ce constat semble avoir une portée allant bien au-delà du Cap et de l'Afrique du Sud.

\section{Bibliographie}

Baker L., 2011. Governing Electricity in South Africa: Wind, Coal and Power Struggles. Norwich (UK), University of East Anglia (Paper 015).

Bridge, G., Bouzarovski S., Bradschaw M., N. Eyre, 2013. Geographies of Energy Transition: Space, Place and the Low-carbon Economy. Energy Policy 53, 331-340.

Bulkeley H., Castan Broto V., Hodson M. Marvin S. (eds), 2011. Cities and Low Carbon Transitions. Routledge, London \& New York.

Bulkeley H., Castán Broto V., Maassen A., 2014. Low-carbon Transitions and the Reconfiguration of Urban Infrastructure. Urban Studies 51 (7) 1471-1486.

Capello R., Nijkamp P., Pepping G., 1999. Sustainable Cities and Energy Policies. Springer Verlag, Berlin and Heidelberg.

Cartwright A., Oelosfe G., Parnell S., Ward S. (eds), 2012. Climate Change and the City Scale. Impacts, mitigation and adaptation in Cape Town. Routledge, London \& New York.

CoCT, 2011. Moving Moutains. Cape Town's Action Plan for Energy and Climate. CoCT, SEA, Cape Town.

CoCT, 2015. Cape Town State of Energy 2015. CoCT, Cape Town.

Coutard, O., 2010. Services Urbains : La Fin des Grands Réseaux ? In O. Coutard, J-P. Lévy (dirs.) Écologies Urbaines. État des Savoirs et Perspectives. Économica-Anthropos, Paris, 102-129.

Christen G., Hamman Ph., 2015. Transition énergétique et inégalités environnementales : Énergies renouvelables et implications citoyennes en Alsace. Presses Universitaires de Strasbourg, Strasbourg.

DoE (Department of Energy), 2015. State of Renewable Energy in South Africa. Republic of South Africa, DoE, Pretoria.

Dubresson A., 2013. À propos d'une initiative municipale verte au Cap (Afrique du Sud) : les leçons du Solar Water Heater Advanced Programme. Flux 93/94, 43-55.

Durand L., Pecqueur B., Senil N., 2015. La transition énergétique par la territorialisation. L'énergie comme ressource territoriale In Scarwell H-J., Leducq D., Groux A. (dirs.), Réussir la transition énergétique, Septentrion, Lille, 29-36. 
Eberhard A., Kolker J., Leigland J., 2014. South Africa's Renewable Energy IPP Procurement Program: Success Factors and Lessons. PPIAF, World Bank Group, Washington DC.

Evrard A., 2013. Contre vents et marées. Politiques des énergies renouvelables. Presses de Sciences Po, Paris.

Evrard A., 2014. Les énergies renouvelables et l'électricité. À propos d'un conflit entre un secteur et une alternative de politique publique. Écologie \& politique 49, 67-80.

Fine B., Rustomjee Z., 1996. The Political Economy of South Africa: From Minerals-Energy Complex to Industrialisation. Westview Press, Boulder (CO).

Froestad J., Shearing C., Herbstein T., Grimwood S., 2012. City of Cape Town Solar Water Heater-Bylaw: Barriers to Implementation In Cartwright A. et al. (eds), Climate Change and the City Scale. Impacts, mitigation and adaptation in Cape Town, Routledge, London \& New York, 244-262.

Gaunt T., 2008. Electricity Distribution Industry Restructuring in South Africa: a case study. Energy Policy 3 6(9), 3448-3459.

Hassenteufel P., 2010. Les processus de mise sur agenda : sélection et construction des problèmes publics. Informations sociales 157 (1), 50-58.

Hodson M., Marvin S., 2010. World Cities and Climate Change: Producing Urban Ecological Security. Open University Press, Maidenhead.

Idasa et al., 2010. Electricity Governance Initiative of South Africa: the governance of Power, Shedding Light on the Electricity Sector in South Africa, Pretoria, Idasa.

Jaglin S., 2014. Urban Energy Policies and the Governance of Multilevel Issues in Cape Town. Urban Studies 51 (7), 1394-1414.

Jaglin S., Dubresson A., 2015a. Eskom. Électricité et pouvoir en Afrique du Sud. Karthala, Paris.

Jaglin S., Dubresson A., 2015b. Sortir de l'addiction au charbon? Transition énergétique et pouvoir d'État en Afrique du Sud In Scarwell H-J., Leducq D., Groux A. (dirs.), Réussir la transition énergétique, Septentrion, Lille, 133-139.

Jaglin S., Verdeil E., 2013. Énergie et villes des pays émergents : des transitions en question. Introduction. Flux 93/94, 7-18.

Jaglin S., Verdeil E. (coords), 2013. Dossier thématique - Énergie et villes des pays émergents : des transitions en question. Flux 93/94.

Jaglin S., Verdeil E., à paraître. Emerging Countries, Cities and Energy: Questioning Transitions, In Bouzarovski S, Pasqualetti M., Castán Broto V. (eds), The Routledge Energy Geographies Companion, Routledge, London \& New York.

Krupa J., Burch S., 2011. A new Energy Future for South Africa; the Political Ecology of South African Renewable Energy. Energy Policy 39, 6254-6261.

Mans U., 2010, "African Cities and Renewable Energy: The Case of Cape Town", 46 ${ }^{\text {th }}$ ISOCARP Congress 2010, Nairobi, Kenya, www.isocarp.net/Data/case_studies/1651.pdf.

McDonald D. (ed.), 2009, Electric capitalism: Recolonising Africa on the power grid. London, Earthscan. HSRC Press, Cape Town.

Monstadt J., Wolff A., 2015. Energy Transition or Incremental Change? Green Policy Agendas and the Adaptability of the Urban Energy Regime in Los Angeles. Energy Policy 78, 213-224.

Morris M., Martin L., 2015. Political Economy of Climate-relevant Policies : the Case of Renewable Energy in South Africa. Institute of Development Studies/University of Cape Town (Evidence Report $n^{\circ} 128$ ), Cape Town.

Pegels A., 2009. Prospects for Renewable Energy in South Africa: Mobilizing the Private Sector. Deutsches Institut für Entwicklungspolitik (Discussion Paper 23), Bonn.

Pickering M., 2010. Towards an Independent System of Operators for South Africa. ERC/UCT, Cape Town.

Roberts D., 2010. Prioritizing Climate Change Adaptation and Local Level Resilience in Durban, South Africa. Environment \& Urbanization. 22 (2), 397-413.

Rojey A., 2008. Énergie \& climat: réussir la transition énergétique. Éditions Technip, Paris. 
Rutherford J., Coutard O., 2014. Urban Energy Transition: Places, Processes and Politics of Socio-technical Change. Urban Studies 51 (7), 1353-1377.

Rutherford J., Jaglin S., 2015. Introduction to the Special Issue -Urban Energy Governance: Local Actions, Capacities and Politics. Energy Policy 78, 173-178.

SALGA \& SACN, 2011. Report of the South African Mayor's Conference on Climate Change in Preparation for COP17/CMP7, Johannesburg, SALGA-SACN, http://sacitiesnetwork.co.za/wp-content/uploads/2014/06/ South-African-Mayors-Conference-on-Climate-Change.pdf.

SEA, 2015. State of Energy in South African Cities, Cape Town, SEA.

Simon D., Leck H., 2015. Understanding Climate Adaptation and Transformation Challenges in African cities. Environmental Sustainability 13, 109-116.

Southall R., 2010. South Africa 2010: From Short-Term Success to Long-Term Decline? In Daniel J., Naidoo P., Pillay D., Southall R., (eds), New South African Review 1: 2010 : Development or Decline? 1-21.

Statistics South Africa, 2015. Financial Census of Municipalities for the Year Ended 30 June 2015. Pretoria, Stats SA.

Taylor A., Cartwright A., Sutherland C. 2014. Institutional Pathways for Local Climate Adaptation: a Comparison of Three South African Municipalities. AFD (Focales 18), Paris.

Theys J., Vidalenc E., 2013. Repenser les villes dans la société postcarbone. Ministère de l'Écologie, du Développement Durable et de l'Énergie (Mission prospective) et ADEME (Service Économie et prospective), Paris.

Thorp B., Marvin S., 1995. Local authorities and energy markets in the 1990s: getting back into power? Local Government Studies 21 (3), 461-482.

Ward, S., Walsh, V., 2010. Cape Town Energy Case Study - City of Cape Town: Energy for Large Cities Report. World Energy Congress 2010. World Energy Council, London. 